\title{
Assessment of Intraoperative Liver Deformation During Hepatic Resection: Prospective Clinical Study
}

\author{
Oleg Heizmann · Stephan Zidowitz • Holger Bourquain • \\ Silke Potthast · Heinz-Otto Peitgen - Daniel Oertli • \\ Christoph Kettelhack
}

Published online: 7 April 2010

(C) Société Internationale de Chirurgie 2010

\begin{abstract}
Background The implementation of intraoperative navigation in liver surgery is handicapped by intraoperative organ shift, tissue deformation, the absence of external landmarks, and anatomical differences in the vascular tree. To investigate the impact of surgical manipulation on the liver surface and intrahepatic structures, we conducted a prospective clinical trial.

Methods Eleven consecutive patients [4 female and 7 male, median age $=67$ years $\quad($ range $=54-80)]$ with malignant liver disease [colorectal metastasis $(n=9)$ and hepatocellular cancer $(n=2)]$ underwent hepatic resection. Pre- and intraoperatively, all patients were studied by CT-based 3D imaging and assessed for the potential value of computer-assisted planning. The degree of liver deformation was demonstrated by comparing pre- and intraoperative imaging.

Results Intraoperative CT imaging was successful in all patients. We found significant deformation of the liver. The deformation of the segmental structures is reflected by the observed variation of the displacements. There is no rigid alignment of the pre- and intraoperative organ positions due to overall deflection of the liver. Locally, a rigid
\end{abstract}

O. Heizmann $(\bowtie) \cdot$ D. Oertli · C. Kettelhack

Department of Surgery, University Hospital Basel,

4031 Basel, Switzerland

e-mail: oheizmann@uhbs.ch

S. Zidowitz · H. Bourquain · H.-O. Peitgen

Fraunhofer MEVIS, Institute for Medical Image Computing,

Bremen, Germany

S. Potthast

Department of Radiology, University Hospital Basel,

Basel, Switzerland alignment of the anatomical structure can be achieved with less than $0.5 \mathrm{~cm}$ discrepancy relative to a segmental unit of the liver. Changes in total liver volume range from -13 to $+24 \%$, with an average absolute difference of $7 \%$.

Conclusions These findings are fundamental for further development and optimization of intraoperative navigation in liver surgery. In particular, these data will play an important role in developing automation of intraoperative continuous registration. This automation compensates for liver shift during surgery and permits real-time 3D visualization of navigation imaging.

\section{Introduction}

Over the last two decades computer-aided diagnosis and intervention planning has gained increasing interest. For example, there has been substantial progress in virtual planning and navigation in neurosurgery $[1,2]$, maxillofacial surgery, and orthopedic surgery [3, 4]. In these fields the transfer of planning information to the intraoperative situation benefits notably from the existence of a consistent osseous reference frame. Such a stable reference frame is not present in visceral surgery due to significant organ deformation and mobility during the intervention. Nevertheless, software-assisted surgical planning based on preoperative imaging plays an increasing role in the evaluation of resectability [5-8], especially in liver surgery.

Liver resection has become increasingly safe as a result of considerable progress in equipment, technology, perioperative management, and surgical technique [9-11]. The main priorities of oncologic liver surgery are complete tumor removal with an appropriate safety margin (i.e., R0 resection) and preservation of a sufficient amount of liver tissue to sustain hepatic function. Because the reasons for 
liver failure after hepatic resection are multifactorial, including underlying liver disease, intraoperative blood loss, or postoperative septic complications, the minimum amount of remaining liver tissue to preserve liver function is difficult to predict. Resection of up to $80 \%$ of total liver volume can be tolerated, provided that there is no impairment of blood supply and venous drainage of the entire remnant liver tissue $[12,13]$. Current classification of liver resections refers to the functional anatomy of the liver [14-16]. Couinaud's model [15] of eight independent portal venous segments and four hepatic venous sectors is schematically based on a regular portal venous and hepatic venous distribution. In contrast, several anatomic studies have shown a different number and size of independent portal segments and hepatic venous sectors, and a great variation in their distribution on the liver surface $[5,17]$.

Preoperative planning of liver resection is based on ultrasound (US), computed tomography (CT), and/or magnetic resonance imaging (MRI) and provides visualization of liver anatomy [18]. While these imaging methods provide excellent visualization of intrahepatic vascular branches, neither the number and distribution nor the extent of liver territories can be assessed reliably. Accordingly, prediction of the remaining and fully vascularized liver parenchyma is inaccurate.

To overcome these shortcomings, software has recently been introduced to enhance the image-based preoperative planning. These methods allow the surgeon to perform virtual liver resections under realistic anatomic conditions, including an automatic calculation of the remnant size and the resection's impact on blood supply and drainage in the remaining liver tissue. The areas at risk for devascularization can be assessed for each dissected vessel separately. The improving impact of software-assisted planning for surgery of hepatobiliary cancers has been reported [6, 8, 19-21].

However, this anatomic information based on threedimensional (3D) reconstruction differs from the intraoperative situation because of the natural mobility, flexibility, and deformation of the liver during mobilization for resection [22, 23]. Intraoperative ultrasound (IOUS) has been used routinely to locate the tumor and as a guide for liver resection. However, there is still difficulty in understanding the $3 \mathrm{D}$ relationship between the tumor and the vascular structures based on two-dimensional (2D) imaging of a small part of the liver limited by information provided by IOUS.

In this article we present the visualization of liver deformation during open abdominal hepatic resections using an intraoperative multislice CT and compare it with the preoperative data. Doing this we were able to document and quantify organ deformation, which is fundamental for further development and optimization of intraoperative navigation in liver surgery.

\section{Materials and methods}

From December 2006 to September 2008, 11 consecutive patients with liver malignancies were included in a prospective clinical phase I study. There were 4 female and 7 male patients with a median age of 67 years (range $=54$ 80). Underlying malignancies were metastasis of colorectal cancer $(n=9)$ and hepatocellular cancer $(n=2)$. Preoperatively, all patients were studied by CT-based 3D imaging and assessed for the potential value of computerassisted planning. The degree of liver deformation was demonstrated and quantified by comparing preoperative and intraoperative imaging.

The study was approved by the local ethics committee and written informed consent was obtained from each patient.

\section{Preoperative setting}

The objective of preoperative multidetector $\mathrm{CT}$ is to display the arterial, portal venous, and hepatic venous anatomy to help identify important vascular variants, to allow volumetric measurements of the whole liver as well as of the remnant liver, and to detect unexpected focal or diffuse liver disease. The preoperative CT imaging protocol, performed with a 64-row multidetector CT (MDCT) scanner (SOMATOM Sensation 64, Siemens Medical Solutions, Erlangen, Germany), consisted of obtaining three image sets after intravenous injection of $120 \mathrm{ml}$ of a nonionic contrast agent (Ultravist 300, Schering, Germany) at a rate of $4 \mathrm{ml} / \mathrm{s}$ with the following parameters: $120 \mathrm{kV}$, $140-170 \mathrm{~mA} \mathrm{~s}$, section thickness/collimation $=16 / 1.5 \mathrm{~mm}$, pitch $=1$, rotation time $=0.5 \mathrm{~s}$, reconstruction interval $=$ $3 \mathrm{~mm} / 1.5 \mathrm{~mm}$. To ensure accuracy, the timing of the arterial phase was determined with real-time bolus tracking in the abdominal aorta at the level of the diaphragm, with a detection threshold of 100 Hounsfield units (HU). Arterial phase imaging was started with a 15-s delay and portalvenous phase imaging with a 45 -s delay.

Analysis of the image data was based on source images as well as on 2D multiplanar reformatted images and 3D MIP images created on a commercially available workstation (Leonardo, Siemens Medical Solutions, Erlangen, Germany). Moreover, anatomical risk analysis and virtual resection planning were conducted for each patient using a dedicated software assistant (MeVis Liver-Viewer, Fraunhofer MEVIS, Bremen, Germany).

Intraoperative setting

The surgical interventions were performed in a "multifunctional image-guided therapy suite"(MIGTS) established at our hospital [24], which has a sterile environment 
and might be used as an imaging and image-guided therapy suite at the same time. A newly developed hybrid imaging/ operation (OR) table (Vascular Interventional Workplace for Advanced Surgery VIWAS, Maquet, Rastatt, Germany) transports the patient and from the OR table onto a receiving $\mathrm{CT}$ table without actual patient transfer.

All patients underwent surgery by an abdominal approach. The surgical procedure began with exploration of abdominal cavity for additional pathological findings. After complete mobilization and exposure of the liver according to the final operation position (Fig. 1), CT imaging was performed under aseptic conditions. To avoid artifacts from metallic devices, a carbon retractor was placed for retraction of wound margins. Prior to parenchyma transection, IOUS was performed routinely to locate the tumor and as a guide for liver resection.

The intraoperative CT imaging protocol, performed with a 10-row MDCT scanner (SOMATOM Sensation 10; Siemens Medical Solutions, Erlangen, Germany), consisted of a portal-venous phase at a rate of $4 \mathrm{ml} / \mathrm{s}$ with the following parameters: $120 \mathrm{kV}, 140170 \mathrm{~mA} \mathrm{~s}$, section thickness/collimation $=16 / 1.5 \mathrm{~mm}$, pitch $=1$, rotation time $=0.5 \mathrm{~s}$, reconstruction interval $=3 \mathrm{~mm} / 1.5 \mathrm{~mm}$. To ensure accuracy, the timing of the portal-venous phase was determined with a real-time bolus tracking in the abdominal aorta at the level of the diaphragm, with a detection threshold of $100 \mathrm{HU}$. Portal-venous phase imaging was started with a 60-s delay.

Image analysis and virtual operation planning

The MDCT data were transferred to the Fraunhofer Institute for Medical Image Computing (MEVIS, Bremen, Germany). The software assistant MeVis-Liver Analyzer was used for image processing and virtual surgical planning. This tool is a stand-alone application dedicated to image-based computer assistance in liver surgery [25-29]. The software offers a standardized workflow for computation of functional liver anatomy and volumetric risk analysis based on the standard 2D CT images. In evaluating the computed remnant liver volume and considering the prospective liver parenchyma with postoperatively impaired blood flow, an optimized resection plan was developed. The results of the preoperative analysis were sent back to the hospital via the internet.

The intraoperative MDCT data were processed likewise at MEVIS to extract the functional liver anatomy and to compute the individual vascular territories. Independent of the preoperative planning, optimized resection plans were developed from the intraoperative data and the results were compared with the preoperative surgical planning.

For a more detailed evaluation of organ deformation, the corresponding anatomical positions were identified semiautomatically in related preoperative and intraoperative data. In addition to characteristic points on the surface of the liver, bifurcations of the vasculature are used to map the inner structure of the organ. In matching these positions, a smoothed deformation field was computed for each patient to estimate the intraoperative displacement and deformation of the organ.

\section{Results}

Intraoperative CT imaging was successful in all patients with common diagnostic quality (Figs. 1 and 2). We observed an increase in operation time of $30 \pm 7$ min due to intraoperative CT scanning. The operations performed included segmentectomy $(n=3)$, bisegmentectomy $(n=$ $1)$, trisegmentectomy $(n=1)$, left hepatectomy $(n=3)$, right hepatectomy $(n=2)$, and extended right hepatectomy $(n=1)$. In only one case did we have to change from the planned right hepatectomy to a bisegmentectomy after assessment by intraoperative ultrasound and intraoperative CT.

The postoperative course was uneventful in ten cases. After a right hepatectomy one patient developed ascites, which resolved with conservative treatment within
Fig. 1 Comparison of preoperative (a) vs. intraoperative (b) situation demonstrates global 2D deformation of hepatic parenchyma
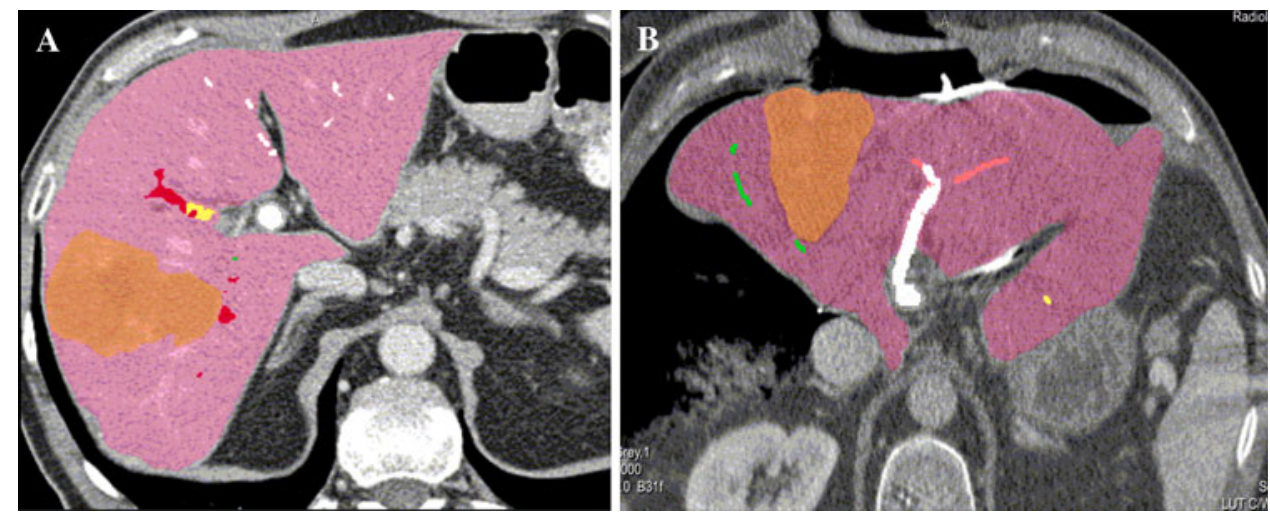
Fig. 2 a Liver with a centrally located colorectal metastasis. Computer-assisted risk analysis with respect to hepatic veins. b Preoperative simulation of a central resection
A

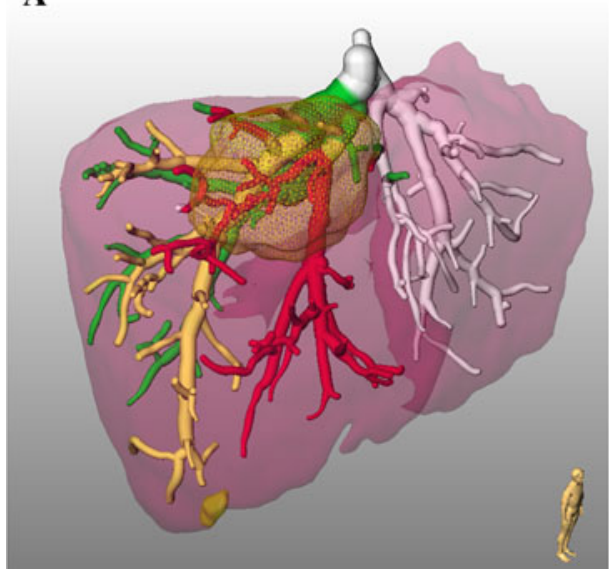

B

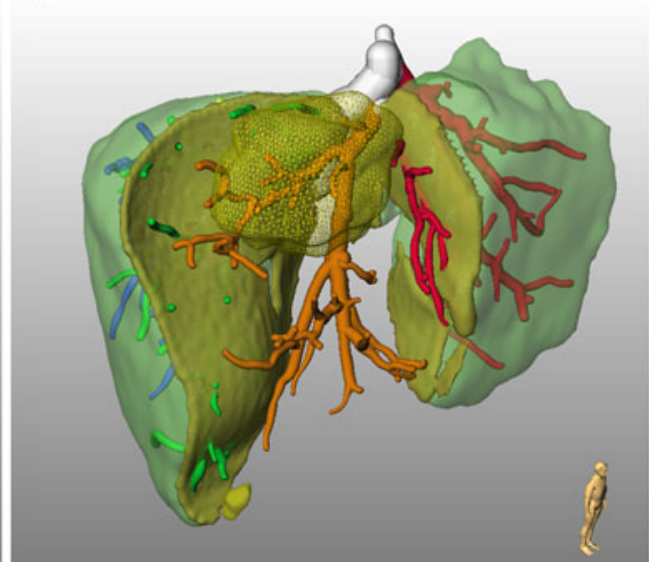

14 days. Histological examination revealed no significant cirrhosis and/or fibrosis of the liver tissue and safety resection margins (R0 resection) in all cases.

Given the careful intraoperative mobilization of the liver, a distinct nonrigid deformation (deviance more than $2 \mathrm{~cm}$ ) of the organ was observed in only about half of the cases. There was no significant difference in deformation with respect to patient age and size and type of the tumor, but the intraoperative deformation was enforced mainly by the bedding of the mobilized organ, e.g., the deformation was caused by external and gravitational forces to overcome the effects of stiffness of the individual organ. Looking at the deformation in detail, some general patterns were observed: The most prominent deformation stemmed from the relocation of the distal left and right parts of the liver due to the intraoperative bedding. Segments P2 and P3 as well as the inferior parts of segments P5 and P6 are either shifted proximal or are driven apart while the organ is flattened out. In relation to a rigid alignment of the central vasculature around the portal vein bifurcation, maximal displacements of the parenchyma position of about $6 \mathrm{~cm}$ were observed in the intraoperative data (Fig. 3).

Changes in total volume of the liver ranged from -13 to $+24 \%$, with an average absolute difference of about $7 \%$ (Table 1). All calculated overall liver volume and resection volume preoperatively and intraoperatively and specimen weight are given in Table 2 . In four patients, the calculated liver volume increased intraoperatively; in the other patients the volume decreased. The relative volumes of the portal-venous vascular territories, as calculated by the ratio of territorial volume to total liver volume, showed good agreement between the preoperative and intraoperative data. The preoperatively and intraoperatively calculated relative territorial volumes differed on average by less than 3\%. The observed outlier (change in mapping from P6 to P7 of $14 \%$ in one case and change of mapping from P5 and P7 to P8 of $10 \%$ in one case) can be explained by the

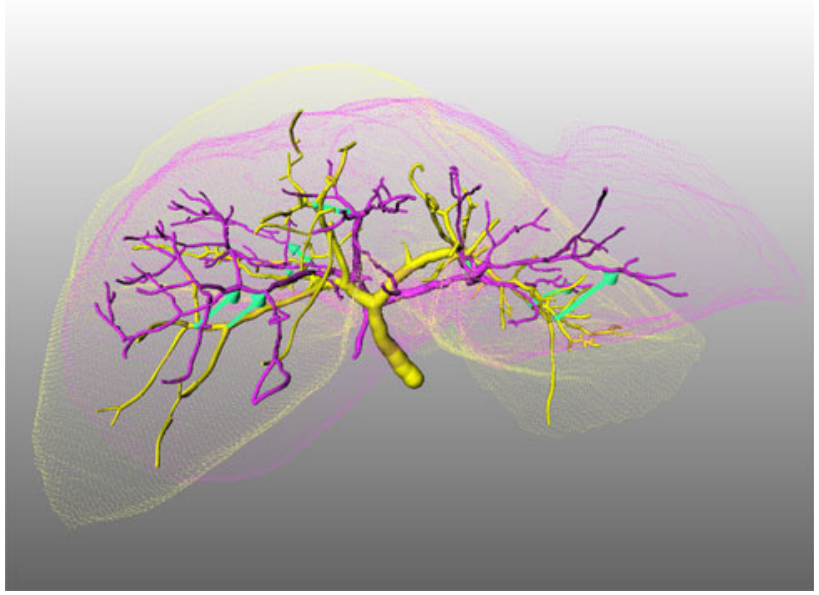

Fig. 3 Comparison of preoperative (yellow) vs. intraoperative (magenta) situation demonstrates impressively the 3D deformation of liver surface and vascular tree. (Color figure online)

poorly contrasted portal vein in the intraoperative images, not allowing for detailed vascular segmentation and thus inaccurate calculation of vascular territories [28], and was not caused by the overall deformation.

This homogeneous deformation of the segmental structure of the liver is reflected by the observed smooth variation of the displacements: While there is no rigid alignment of the pre- and intraoperative organ positions due to the observed overall deflection of the organ, locally a rigid alignment of the anatomical structure can be achieved with less than $0.5 \mathrm{~cm}$ discrepancy relative to a segmental unit of the liver.

\section{Discussion}

The aim of our study was to determine the dimension and quantity of liver deformation caused by natural mobility, flexibility, and surgical mobilization for resection. To our 
Table 1 Overall difference of liver volume preoperatively vs. intraoperatively and of particular portal-venous territories

\begin{tabular}{|c|c|c|c|c|c|c|c|c|c|c|}
\hline \multirow[t]{2}{*}{ Case } & \multirow{2}{*}{$\begin{array}{l}\text { Overall volume } \\
\text { difference }(\%)\end{array}$} & \multicolumn{9}{|c|}{ Stability of portal-venous territories (\%) } \\
\hline & & P1 & $\mathrm{P} 2$ & P3 & $\mathrm{P} 4 \mathrm{a}$ & $\mathrm{P} 4 \mathrm{~b}$ & P5 & P6 & P7 & P8 \\
\hline 1 & -2.58 & 0.00 & 9.13 & -9.95 & 1.33 & 0.00 & 0.17 & -0.53 & -4.04 & 3.82 \\
\hline 2 & 10.88 & -0.07 & -0.57 & 0.54 & 1.34 & -0.61 & -0.03 & -0.41 & -2.22 & 2.17 \\
\hline 3 & -6.36 & 0.68 & 2.88 & 0.37 & -0.18 & -1.01 & -2.17 & 2.59 & -2.21 & -0.89 \\
\hline 4 & 23.99 & -0.28 & 0.17 & 1.58 & -0.46 & 1.20 & 3.01 & -1.81 & -2.43 & -1.05 \\
\hline 5 & -6.86 & -4.57 & 6.62 & 7.79 & -4.33 & -6.39 & 4.60 & -2.08 & -5.07 & 3.28 \\
\hline 6 & 7.36 & -0.97 & 0.96 & 2.51 & 2.27 & -1.27 & 0.00 & 13.35 & -16.85 & 0.00 \\
\hline 7 & -0.26 & 0.61 & 0.35 & 0.68 & 0.14 & -0.46 & 0.09 & -1.02 & -1.76 & 1.31 \\
\hline 8 & -12.62 & -0.51 & -0.58 & 1.90 & -0.14 & -0.05 & 0.31 & -1.79 & -0.40 & 1.32 \\
\hline 9 & -4.31 & 2.48 & -2.60 & 0.00 & 0.43 & 1.94 & 0.31 & 0.58 & -1.29 & -2.13 \\
\hline 10 & 5.49 & 3.88 & -1.88 & -2.32 & 0.90 & 4.87 & -8.54 & -0.02 & -6.29 & 9.38 \\
\hline 11 & -6.83 & -1.92 & -3.19 & 7.95 & 4.31 & -3.77 & 1.77 & 0.18 & -0.13 & -5.31 \\
\hline
\end{tabular}

Gray entries are nonvalid data because of nonoptimal contrasting of the vessels (error in overall volume difference $= \pm 5 \%$; error in portalvenous territory changes $= \pm 2 \%$ )

Table 2 Calculated overall liver volume and resection volume preoperatively and intraoperatively and specimen weight

Relative error in volume accuracy $= \pm 4 \%$

\begin{tabular}{llclcl}
\hline $\begin{array}{l}\text { Case } \\
\text { pre-OP CT (ml) }\end{array}$ & $\begin{array}{l}\text { Liver volume osection volume on } \\
\text { pre-OP CT [ml (\%)] }\end{array}$ & $\begin{array}{l}\text { Liver volume on } \\
\text { intra-OP CT (ml) }\end{array}$ & $\begin{array}{l}\text { Resection volume on } \\
\text { intra-OP CT [ml (\%)] }\end{array}$ & $\begin{array}{l}\text { Resection } \\
\text { weight }(\mathrm{g})\end{array}$ \\
\hline 1 & 1316 & $210(16)$ & 1282 & $197(15)$ & n.d. \\
2 & 1213 & $287(24)$ & 1345 & $307(23)$ & 340 \\
3 & 1698 & $376(22)$ & 1590 & $401(25)$ & 400 \\
4 & 1530 & $85(06)$ & 1897 & $91(05)$ & 95 \\
5 & 1356 & $120(12)$ & 1263 & $122(10)$ & 214 \\
6 & 1644 & $1157(70)$ & 1765 & $1022(58)$ & 930 \\
7 & 1518 & $71(05)$ & 1514 & $79(05)$ & 100 \\
8 & 1521 & $1044(69)$ & 1329 & $981(74)$ & .d. \\
9 & 1815 & $67(04)$ & 1731 & $88(05)$ & 71 \\
10 & 856 & $124(14)$ & 903 & $131(14)$ & 125 \\
11 & 1874 & $1298(69)$ & 1746 & $1076(62)$ & 995 \\
\hline
\end{tabular}

knowledge, this is the first description of entire deformation following surgical mobilization of the liver.

Nowadays, preoperative planning in liver surgery is based on US, CT, and/or MRI with excellent visualization of liver anatomy [18]. While these imaging methods provide good visualization of the intrahepatic vascular tree, neither the number and distribution nor the extent of vascular territories of the liver can be assessed satisfactorily. Accordingly, prediction of the remaining and fully vascularized liver tissue is inaccurate. To overcome these shortcomings, software programs have been introduced recently to improve image-based preoperative planning [25]. These methods allow the surgeon to perform virtual liver resections under realistic anatomic conditions, including an automatic calculation of the remnant size, the resection's impact on blood supply, and drainage in the remaining liver tissue. The areas at risk for devascularization can be assessed for each dissected vessel separately. The improving impact of the software-aided planning for hepatobiliary cancer surgery has been reported $[6,8,19-$ 21]. These data raise the question of whether 3D-based preoperative planning can be a fundamental part of intraoperative navigation during liver resection. However, the anatomic information from 3D reconstruction differs from the intraoperative situation because of natural mobility, flexibility, and deformation of the liver due to mobilization for resection [23]. To compensate for these factors, three different categories of studies involving image-guided liver surgery have been published. The first category consists of registration based on the geometric features of the liver. Concordant features between the data sets were identified and matched by minimizing a distance measure between 
two sets $[22,30,31]$. The second category uses the complex vascular tree to perform the registration between preoperative images and the intraoperative ultrasound data [32-34]. The third type of registration is intensity based, where a correlation measure between two image sets is maximized. This method requires intraoperative ultrasonographic or tomographic data [32, 33, 35-37]. However, there is still no published detailed information about the impact of surgical manipulation during hepatic resection with respect to the deformation of the liver surface and intrahepatic structures. This would be required for the development of an exact and user-friendly navigation system.

To investigate the effect of surgical manipulation on the dislocation of the surface and intrahepatic structures and on the volume of the liver during hepatic resection, we performed this prospective clinical trial. The observed homogeneous deformation of the segmental structure of the liver implies that the relative proportions of the segmental volumes stay in good accordance with the preoperatively calculated relationships. However, the total volume of the organ may change significantly. The virtual resection planning based on the intraoperative data is in good alignment with the preoperative deduced surgical plan.

Due to the prominent intraoperative deformation of the organ, no overall rigid alignment of the preoperative planning data with the intraoperative situation which satisfactorily covers the whole organ can be achieved. To use planning information based on preoperative MDCT data for intraoperative navigation, the manifest nonrigid deformation of the organ must be taken into account. A solely rigid alignment of the preoperative spatial information, e.g., by using anatomical landmarks on the surface of the liver, will result in a significant misalignment of anatomical positions by several centimeters. However, looking not at the organ in total but concentrating on a more localized situation (e.g., the area surrounding the planned resection path), the situation seems more promising.

With careful and controlled mobilization and intraoperative bedding of the organ, the observed isotropic deformation of the parenchyma suggests that a rigid alignment of the preoperative data can give satisfactory accuracy for the local navigation. Such alignment should be adjusted by following the local vasculature in an appropriate manner. We presume that in the future intraoperative registration for adjustment to preoperative 3D imaging and operation planning should be preferentially performed by tracked intraoperative ultrasound using the surface and the vascular tree of the liver. The intraoperative CT scan also provides useful data and is a valuable tool for performing complete and safe resection in special cases with central tumors. However, because it is very time consuming, we do not use intraoperative $\mathrm{CT}$ for routine surgery. Furthermore, only a few hospitals around the world have a multifunctional image-guided therapy suite (MIGTS) as is available at our institution [24].

From our point of view, the present findings are fundamental for further developments and optimization of intraoperative navigation in liver surgery, particularly the intraoperative automation of continuous re-registration to compensate for progressive liver shift during surgery and the real-time 3D visualization of navigation imaging.

\section{References}

1. Auer LM, Auer DP (1998) Virtual endoscopy for planning and simulation of minimally invasive neurosurgery. Neurosurgery 43:529-537

2. Kockro RA, Serra L, Tseng-Tsai Y et al (2000) Planning and simulation of neurosurgery in a virtual reality environment. Neurosurgery 46:118-135

3. Chao EY, Barrance P, Genda E et al (1997) Virtual reality (VR) techniques in orthopaedic research and practice. Stud Health Technol Inform 39:107-114

4. Sutherland CJ (1986) Practical application of computer-generated three-dimensional reconstructions in orthopedic surgery. Orthop Clin North Am 17:651-656

5. Fischer L, Cardenas C, Thorn M et al (2002) Limits of Couinaud's liver segment classification: a quantitative computer-based three-dimensional analysis. J Comput Assist Tomogr 26:962-967

6. Lamade W, Glombitza G, Fischer L et al (2000) The impact of 3-dimensional reconstructions on operation planning in liver surgery. Arch Surg 135:1256-1261

7. Marescaux J, Clement JM, Tassetti V et al (1998) Virtual reality applied to hepatic surgery simulation: the next revolution. Ann Surg 228:627-634

8. Oldhafer KJ, Hogemann D, Stamm G (1999) 3-dimensional (3-D) visualization of the liver for planning extensive liver resections. Chirurg 70:233-238

9. Heizmann O, Loehe F, Volk A (2008) Ischemic preconditioning improves postoperative outcome after liver resections: a randomized controlled study. Eur J Med Res 13:79-86

10. Rau HG, Schauer R, Helmberger $T$ et al (2000) Impact of virtual reality imaging on hepatic liver tumor resection: calculation of risk. Langenbecks Arch Surg 385:162-170

11. Schmidbauer S, Hallfeldt KK, Sitzmann G (2002) Experience with ultrasound scissors and blades (UltraCision) in open and laparoscopic liver resection. Ann Surg 235:27-30

12. Vauthey JN, Chaoui A, Do KA et al (2000) Standardized measurement of the future liver remnant prior to extended liver resection: methodology and clinical associations. Surgery 127:512-519

13. Yigitler C, Farges O, Kianmanesh R et al (2003) The small remnant liver after major liver resection: how common and how relevant? Liver Transpl 9:S18-S25

14. Bismuth H (1982) Surgical anatomy and anatomical surgery of the liver. World J Surg 6:3-9

15. Couinaud C (1954) Lobes et Segments Hepatiques - Notes Sur Larchitecture Anatomique et Chirurgicale du Foie. Presse Med 62:709-712

16. Strasberg SM (2005) Nomenclature of hepatic anatomy and resections: a review of the Brisbane 2000 system. J Hepatobiliary Pancreat Surg 12:351-355 
17. Lang H, Junge A, Sitter H et al (1995) Liver segmentectomy as anatomically precise resections. An experimental study in sheep. Eur J Surg 161:677-682

18. Wigmore SJ, Redhead DN, Yan XJ et al (2001) Virtual hepatic resection using three-dimensional reconstruction of helical computed tomography angioportograms. Ann Surg 233:221-226

19. Endo I, Shimada H, Sugita M et al (2007) Role of three-dimensional imaging in operative planning for hilar cholangiocarcinoma. Surgery 142:666-675

20. Lang H, Radtke A, Hindennach $M$ et al (2005) Impact of virtual tumor resection and computer-assisted risk analysis on operation planning and intraoperative strategy in major hepatic resection. Arch Surg 140:629-638

21. Saito S, Yamanaka J, Miura K et al (2005) A novel 3D hepatectomy simulation based on liver circulation: application to liver resection and transplantation. Hepatology 41:1297-1304

22. Cash DM, Miga MI, Glasgow SC et al (2007) Concepts and preliminary data toward the realization of image-guided liver surgery. J Gastrointest Surg 11:844-859

23. Hayashibe M, Suzuki N, Kobayashi S et al (2005) Development of a 3D visualization system for surgical field deformation with geometric pattern projection. Stud Health Technol Inform 111:172-177

24. Jacob AL, Regazzoni P, Steinbrich W et al (2000) The multifunctional therapy room of the future: image guidance, interdisciplinarity, integration and impact on patient pathways. Eur Radiol 10:1763-1769

25. Bourquain H, Schenk A, Link F et al (2002) HepaVision 2: a software assistant for preoperative planning in living-related liver transplantation and oncologic liver surgery. In: Lemke HU, Vannier MW, Inamura K, Farman AG, Doi K, Reiber JHC (eds) CARS 2002: Computer Assisted Radiology and Surgery: Proceedings of the 16th International Congress and Exhibition, Paris, June 26-29, 2002. Springer-Verlag, New York, pp 341-346

26. Preim B, Bourquain H, Selle D et al (2002) Resection proposals for oncologic liver surgery based on vascular territories. In: Lemke HU, Vannier MW, Inamura K, Farman AG, Doi K, Reiber JHC (eds), CARS 2002: Computer Assisted Radiology and Surgery: Proceedings of the 16th international congress and exhibition, Paris, June 26-29, 2002, Springer-Verlag, New York, pp 353-358
27. Schenk A, Prause G, Peitgen HO (2000) Efficient semiautomatic segmentation of 3D objects. In: Delp SL, DiGioia AM, Jaramaz B (eds) Medical Image Computing and Computer-Assisted Intervention: MICCAI 2000, Proceedings of the third international conference, Pittsburgh, PA, USA, October 11-14, 2000. Lecture Notes in Computer Science, vol 1935, pp 186-195

28. Selle D, Preim B, Schenk A et al (2002) Analysis of vasculature for liver surgical planning. IEEE Trans Med Imaging 21:13441357

29. Van Ooijen PMA, Wolf R, Schenk A et al (2003) Recent developments in organ-selective reconstruction and analysis of multiphase liver CT. Imaging Decis 7:37-43

30. Herline AJ, Herring JL, Stefansic JD et al (2000) Surface registration for use in interactive, image-guided liver surgery. Comput Aided Surg 5:11-17

31. Voirin D, Payan Y, Amavizca M et al (2002) Computer-aided hepatic tumour ablation: requirements and preliminary results. $\mathrm{C}$ R Biol 325:309-319

32. Aylward SR, Cleary KR, Hawkes DJ (2005) Intraoperative image processing for surgical guidance. IEEE Trans Med Imaging 24:1401-1404

33. Porter BC, Rubens DJ, Strang JG et al (2001) Three-dimensional registration and fusion of ultrasound and MRI using major vessels as fiducial markers. IEEE Trans Med Imaging 20:354-359

34. Hassenpflug P, Schobinger M, Vetter M et al (2008) Generation of attributed relational vessel graphs from threedimensional freehand ultrasound for intraoperative registration in image-guided liver surgery. In: Galloway RL (ed) Medical Imaging 2003Visualization, Image-Guided Procedures, and Display. Proceedings of SPIE 5029, pp 222-230

35. Carrillo A, Duerk JL, Lewin JS et al (2000) Semiautomatic 3-D image registration as applied to interventional MRI liver cancer treatment. IEEE Trans Med Imaging 19:175-185

36. Penney GP, Blackall JM, Hamady MS et al (2004) Registration of freehand 3D ultrasound and magnetic resonance liver images. Med Image Anal 8:81-91

37. Wilson DL, Carrillo A, Zheng L et al (1998) Evaluation of 3D image registration as applied to MR-guided thermal treatment of liver cancer. J Magn Reson Imaging 8:77-84 